Quim. Nova, Vol. 34, No. 3, 414-418, 2011

\title{
AUMENTO DA RESPOSTA ANALÍTICA POR MEIO DA OTIMIZAÇÃO DO SISTEMA DE INJEÇÃO SEM DIVISÃO DE FLUXO EM CROMATOGRAFIA GASOSA EMPREGANDO A LEI DOS GASES IDEAIS
}

\author{
Guilherme Post Sabin, Osmar Damian Prestes, Manoel Leonardo Martins, Martha Bohrer Adaime e Renato Zanella* \\ Departamento de Química, Universidade Federal de Santa Maria, 97105-900 Santa Maria - RS, Brasil
}

Recebido em 25/5/10; aceito em 27/10/10; publicado na web em 26/1/11

\begin{abstract}
ANALYTICAL RESPONSE ENHANCEMENT THROUGH THE OPTIMIZATION OF THE SPLITLESS INJECTION SYSTEM IN GAS CHROMATOGRAPHY BY EMPLOYING THE IDEAL GAS LAW. This paper reports the development of an easy, fast and effective procedure for the verification of the ideal gas law in splitless injection systems in order to improve the response. Results of a group of pesticides were used to demonstrate the suitability of the approach. The procedure helps establish experimental parameters through theoretical aspects. The improved instrumental response allowed extraction with lower sample volumes, the minimization of time and costs and the simplification of sample preparation.
\end{abstract}

Keywords: chromatographic methods; splitless inlet; backflush volume.

\section{INTRODUÇÃO}

Em análises ambientais e na área de saúde, o limite de quantificação surge como desafio imposto pelos baixos níveis de concentração dos analitos de interesse. No caso dos pesticidas, por exemplo, a quantificação de resíduos em amostras ambientais decorrentes da utilização destes compostos requer a utilização de métodos que permitam atingir limites de quantificação adequados. Portanto, a necessidade de monitorar estas substâncias em concentrações cada vez menores tem exigido um esforço adicional na busca por melhores limites de quantificação. ${ }^{1}$ Os limites mais baixos permitidos pela legislação brasileira para pesticidas em água potável ${ }^{2}$ e de superfície ${ }^{3}$ são 30 e $1 \mathrm{ng} \mathrm{L}^{-1}$, respectivamente, e dependem principalmente do grau de toxicidade de cada pesticida.

Neste sentido, uma das técnicas mais utilizadas para análise de compostos orgânicos, suficientemente voláteis e termicamente estáveis, tem sido a cromatografia gasosa acoplada a diferentes sistemas de detecção, ${ }^{4,5}$ com destaque para a espectrometria de massas $(\text { GC-MS })^{6,7}$ que permite a determinação simultânea de vários compostos de diversas classes.$^{8-10}$ Nas determinações cromatográficas as propriedades físico-químicas dos analitos devem ser consideradas para os métodos obterem alta seletividade e sensibilidade. ${ }^{11,12}$ Por esta razão, a análise instrumental é, geralmente, precedida por um preparo de amostra adequado visando prover tanto seletividade como sensibilidade. ${ }^{13}$ Entretanto, embora estas etapas de extração e préconcentração em aplicações ambientais sejam importantes, são estas etapas que demandam grande parte do tempo da análise, aumentam os custos de análise, geram mais resíduos no laboratório e aumentam a incerteza analítica. ${ }^{14}$ Aspectos estatísticos sobre o preparo de amostras foram descritos por Wells. ${ }^{12}$

Uma alternativa viável para minimizar esforços em busca de fatores de pré-concentração elevados é a otimização dos parâmetros cromatográficos, ${ }^{13}$ em particular, do sistema de injeção pulsado. ${ }^{15}$ Nas publicações de Grob ${ }^{16}$ e Grob e Barry ${ }^{17}$ os sistemas de injeção sem divisão de fluxo (splitless) são abordados extensivamente. Um enfoque mais conciso da influência das variáveis termodinâmicas

*e-mail: rzanella@base.ufsm.br presentes em sistemas splitless foi descrito no guia técnico da empresa Restek. ${ }^{18}$ As tentativas de aumento de resposta analítica por meio do aumento de volume injetado, associado com programas de temperatura do injetor, foram apresentadas nos trabalhos de Kirchner et al. ${ }^{19}$ e de Hoh e Mastovska. ${ }^{20}$ Os procedimentos propostos são eficientes para aumentar a sensibilidade, entretanto apresentam algumas desvantagens, como a necessidade de avaliar um grande número de parâmetros de otimização e demandar maior tempo entre as análises.

Este trabalho objetivou demonstrar a aplicabilidade dos fundamentos teóricos da termodinâmica dos gases na otimização do sistema de injeção splitless por meio de uma forma rápida para verificação prática dos modelos otimizados, a partir de um planejamento experimental empregando um modelo quadrático. A otimização permite o aumento de resposta instrumental e, portanto, redução dos fatores de pré-concentração necessários, simplificando o método. Avaliouse, também, a aplicação do método proposto na análise de um grupo de 20 compostos representativos (molinato, trifluralina, hexaclorobenzeno, simazina, atrazina, lindano, propanil, heptacloro, alaclor, aldrin, metolacloro, pendimetalina, heptacloro epóxido, clordano, endossulfan, dieldrin, endrin, DDT, metoxicloro e permetrina) com diferentes comportamentos cromatográficos visando o emprego em análises multirresíduo.

\section{PARTE EXPERIMENTAL}

\section{Materiais e métodos}

Para este estudo foi empregado um cromatógrafo a gás HP 6890N Agilent (Wilmington, Estados Unidos) operado com programação de temperatura multilinear (estágios entre 50 e $270{ }^{\circ} \mathrm{C}$ ), totalizando $29,7 \mathrm{~min}$. O cromatográfico foi equipado com injetor para divisão do fluxo split/splitless, septo com baixo sangramento (5181-3383) e insersor de quartzo silanizado modelo splitless (5062-3587) com volume efetivo de 0,493 mL. O sistema de injeção foi composto por seringa cromatográfica de $10 \mu \mathrm{L}$, Agilent (5181-1267), torre de injeção automática (velocidade de injeção: $\left.6000 \mu \mathrm{L} \mathrm{s}^{-1}\right)$ e amostrador automático para 100 frascos Agilent série 7683 (Wilmington, Estados Unidos). 
Na separação de compostos foi utilizada coluna analítica capilar de baixa polaridade HP5-MS de baixo sangramento, com $30 \mathrm{~m}$ de comprimento, 0,25 mm de diâmetro interno e espessura de filme (fase estacionária) de 0,25 $\mu \mathrm{m}$, Agilent (19091S-433).

Para detecção foi empregado um espectrômetro de massas HP 5973N Agilent (Palo Alto, Estados Unidos), com analisador de massas quadrupolar capaz de varrer de $1-800 \mathrm{~m} / \mathrm{z}$ e fonte de ionização por impacto de elétrons a $70 \mathrm{eV}$, operado a $280^{\circ} \mathrm{C}$.

Nas pesagens uma balança analítica AG285 Mettler Toledo (Zurich, Suíça), com resolução de $0,01 \mathrm{mg}$ (até $81 \mathrm{~g}$ ) e $0,1 \mathrm{mg}$ (até $210 \mathrm{~g}$ ) foi utilizada. As balanças foram verificadas com padrões de massas Throemner certificados pela RBC (Rede Brasileira de Calibração).

Para o preparo das soluções utilizaram-se vidrarias volumétricas Classe A calibradas conforme critérios da norma ISO DIN 1042. Transferidores com capacidade entre 100 e $1000 \mu \mathrm{L}$ Brand e ponteiras foram empregados no preparo de soluções e frascos para amostras $2 \mathrm{~mL}$ em vidro transparente com tampa e septo de teflon, HP, foram utilizados. O solvente utilizado no preparo de soluções foi o acetato de etila PA-ACS 99,5\%, Quimex. O gás utilizado como fase móvel foi o hélio UP 99,999\%, AGA. Maiores detalhes sobre os procedimentos de preparo da amostra, estudo de otimização de separação e validação detalhada, podem ser encontrados em Sabin. ${ }^{14}$

\section{Otimização do sistema de injeção}

Embora os fatores que determinam o aumento de sensibilidade não estejam apenas relacionados ao sistema de injeção, mas também ao sistema de separação e detecção, neste trabalho foram abordados principalmente os parâmetros relacionados ao sistema de injeção e às variáveis termodinâmicas descritas nas leis dos gases ideais. Uma observação mais detalhada sobre a lei dos gases ideais remete-nos a uma breve explicação sobre sua origem. Neste sentido, a lei de Charles estabelece que, sob pressão constante, o volume de uma quantidade constante de gás aumenta proporcionalmente com a temperatura. A lei de Boyle-Mariotte estabelece que, sob transformação isotérmica, o produto entre pressão e volume é constante; e Gay-Lussac afirma que, sob um volume e quantidade de gás constantes, a pressão é diretamente proporcional à temperatura. Por fim, Avogadro acrescenta que o aumento do número de partículas implica no aumento do número de colisões e um igual número de partículas para gases diferentes implica em um igual número de colisões. Assim, as leis de Charles, Boyle-Mariotte Gay-Lussac e Avogadro podem ser expressas pela equação de estado de Clapeyron (equação que reúne as leis descritas acima). ${ }^{21,22}$

Para avaliação das respostas do sistema GC-MS obtidas por meio do planejamento experimental foram empregados 8 compostos representativos (um padrão interno, dois padrões de controle (surrogate) e cinco pesticidas) conforme apresentado na Tabela 1. A programação de temperatura do forno foi otimizada ${ }^{14}$ e consiste em uma rampa de temperatura multilinear. A detecção foi realizada através do modo de monitoramento seletivo de íons no sistema GC-MS. ${ }^{14,23}$ Os demais parâmetros do método são enfatizados na sequência.

O estudo de otimização do sistema de injeção splitless foi efetuado por meio da análise empregando GC-MS para multiclasse de resíduos de pesticidas regulados pela portaria brasileira de potabilidade de água. ${ }^{2}$ A seguir estão descritos os parâmetros e as etapas utilizadas para a otimização do sistema de injeção.

\section{Solvente}

Foram considerados aspectos como: solubilidade dos analitos, toxicidade, custo, ponto de ebulição e massa molar. Neste sentido, o acetato de etila foi considerado a melhor opção de solvente por sua polaridade moderada (adequado para determinação multiclasse de
Tabela 1. Informações dos compostos avaliados

\begin{tabular}{ccccc}
\hline Compostos & Característica & $\begin{array}{c}\text { Concentração } \\
\left(\mu \mathrm{g} \mathrm{L}^{-1}\right)\end{array}$ & $\begin{array}{c}\mathrm{t}_{\mathrm{R}} \\
(\mathrm{min})\end{array}$ & $\begin{array}{c}\mathrm{M} \\
\left(\mathrm{g} \mathrm{mol}^{-1}\right)\end{array}$ \\
\hline 1,4-diclorobenzeno-d4 & surrogate & 40 & 5,1 & 151,0 \\
molinato & pesticida & 25 & 9,7 & 187,3 \\
hexaclorobenzeno & pesticida & 25 & 13,5 & 284,8 \\
antraceno-d10 & surrogate & 40 & 14,8 & 188,3 \\
metolacloro & pesticida & 25 & 18,3 & 283,8 \\
1,3-difenoxibenzeno & padrão interno & 500 & 20,3 & 170,2 \\
DDT & pesticida & 25 & 22,4 & 354,5 \\
permetrina & pesticida & 25 & 25,5 & 391,3 \\
\hline
\end{tabular}

pesticidas), baixa toxicidade, baixo custo, temperatura de ebulição moderada $\left(77^{\circ} \mathrm{C}\right.$, permitindo efeito solvente a $\left.50{ }^{\circ} \mathrm{C}\right)$ e massa molar relativamente alta (possibilitando bom coeficiente de expansão térmica).

\section{Pressão no injetor}

Foram definidos três níveis de pressão $(7,5,15$ e 25 psi). A pressão mais baixa é decorrente da configuração de parâmetros cromatográficos normalmente adotados para o sistema splitless: dimensões da coluna (HP5-MS $30 \mathrm{~m}$ x 0,25 mm x 0,25 $\mu \mathrm{m}$ ), temperatura inicial do forno $\left(50^{\circ} \mathrm{C}\right)$ e hélio como gás de arraste. Para a obtenção dos outros dois níveis de pressão foi utilizado modo pulsado (modo em que, por um período de tempo, a pressão é aumentada no injetor) durante 1,5 min. O pulso de pressão altera a vazão do gás de arraste no período programado ${ }^{24} \mathrm{e}$, portanto, a temperatura inicial do forno deve ser suficientemente baixa para evitar deslocamento nos tempos de retenção $\left(t_{R}\right)$ dos analitos. A ocorrência de alteração nos valores de $t_{R}$ dos compostos demandaria a necessidade de alteração do método utilizado no detector MS. Deve-se considerar que quanto menor for o diâmetro da coluna, ou maior o seu comprimento, maior será a pressão do gás no injetor necessária para obter uma determinada vazão de fase móvel, o que possibilita aumento do volume injetado. ${ }^{25}$

\section{Temperatura do injetor}

As condições de temperatura escolhidas $\left(240,250\right.$ e $\left.260{ }^{\circ} \mathrm{C}\right)$ foram baseadas nas características físico-químicas dos pesticidas de interesse. ${ }^{26}$ A temperatura do injetor deve ser alta o suficiente para vaporizar completamente os analitos, minimizando o tempo de permanência no injetor para evitar a ocorrência de degradação. Entretanto, temperaturas mais baixas que ainda permitam a vaporização dos analitos são preferidas porque reduzem a possibilidade de degradação dos analitos e proporcionam um volume de expansão adequado, minimizando a ocorrência do fenômeno denominado backflush, ${ }^{27}$ que ocorre quando o volume de solvente expandido na fase gasosa excede o volume efetivo do insersor (liner).

\section{Volume de injeção}

Para a escolha do volume de líquido injetado foram utilizados volumes de 1,2 e $3 \mu \mathrm{L}$ que representam condições em torno do volume de backflush do sistema.

\section{Tipo de insersor}

Este é um importante parâmetro associado ao volume de gás suportado pelo insersor, volume de backflush, ou ainda, volume efetivo do insersor $\left(\mathrm{V}_{\mathrm{f}}\right) .{ }^{24}$ Assim, foi utilizado um insersor tipo splitless com aproximadamente $0,490 \mathrm{~mL}$ de volume efetivo, que corresponde à metade do volume total do insersor $\left(\mathrm{V}_{\mathrm{T}}\right)$ obtido pela Equação 1: 


$$
2 \mathrm{~V}_{\mathrm{f}}=\mathrm{V}_{\mathrm{T}}=\mathrm{L} \cdot \pi \cdot \mathrm{r}^{2}
$$

onde: $\mathrm{L}=78 \mathrm{~mm}$ (comprimento do insersor); $\pi=3,1416$ (constante); $\mathrm{r}=2 \mathrm{~mm}$ (raio interno do insersor).

O insersor utilizado proporcionou volumes de injeção maiores e foi silanizado para minimizar a interação dos analitos mais polares com as paredes internas do mesmo.

A seguir, estão descritas as várias etapas dos estudos de otimização realizados.

\section{Primeira etapa}

Elaboração de um planejamento experimental para criação de um modelo quadrático. ${ }^{28} \mathrm{~A}$ escolha do tipo de modelo está baseada na quantidade de fatores que afetam a resposta instrumental de forma independente no modelo. Ou seja, para obter uma explicação adequada de um conjunto de três variáveis independentes foi preciso utilizar um modelo de segunda ordem. As variáveis pressão, temperatura e volume de injeção foram avaliadas em três níveis cada. A Tabela 2 mostra o planejamento das variáveis independentes utilizadas.

Tabela 2. Resumo dos resultados de otimização do sistema de injeção em função das diferentes variáveis do sistema

\begin{tabular}{cccccc}
\hline & $\begin{array}{c}\text { Variáveis } \\
\text { independentes } \\
\begin{array}{c}\text { Pressão } \\
(\mathrm{psi})\end{array}\end{array}$ & $\begin{array}{c}\text { Temperatura } \\
\left({ }^{\circ} \mathrm{C}\right)\end{array}$ & $\begin{array}{c}\text { Valores } \\
\text { Volume } \\
(\mu \mathrm{L})\end{array}$ & $\begin{array}{c}\text { calculados } \\
\text { Volume de gás } \\
(\mu \mathrm{L})\end{array}$ & $\begin{array}{c}\text { Variáveis } \\
\text { dependentes } \\
\text { Área normalizada } \\
\text { dos picos }(\%)\end{array}$ \\
\hline 7,5 & 240 & 1 & 285 & 14,1 & 1,7 \\
Média & Desvio padrão \\
7,5 & 260 & 1 & 297 & 17,3 & 1,5 \\
7,5 & 240 & 3 & 856 & 20,0 & 1,4 \\
15 & 250 & 1 & 217 & 17,2 & 1,7 \\
7,5 & 250 & 2 & 582 & 17,4 & 1,9 \\
15 & 240 & 2 & 427 & 42,5 & 4,3 \\
15 & 250 & 2 & 435 & 58,0 & 7,8 \\
25 & 260 & 3 & 497 & 99,3 & 1,4 \\
25 & 260 & 2 & 332 & 62,4 & 4,3 \\
15 & 260 & 3 & 443 & 54,1 & 2,6 \\
25 & 250 & 3 & 488 & 98,1 & 1,7 \\
25 & 240 & 1 & 160 & 31,3 & 4,9 \\
\hline
\end{tabular}

\section{Segunda etapa}

Cálculo dos volumes de gás obtidos a partir das condições experimentais de cada um dos experimentos apresentados na Tabela 2, segundo a lei dos gases ideais de Charles, Boyle, Gay-Lussac e Avogadro. O cálculo para o volume de expansão foi realizado empregando-se a Equação 2:

$$
\mathrm{V}=\mathrm{mRT} / \mathrm{pM}
$$

onde: $\mathrm{V}=$ volume de expansão do solvente injetado $(\mathrm{mL}) ; \mathrm{m}=$ massa do solvente injetado (g). Como o solvente é injetado em $\mu \mathrm{L}$, converte-se o volume para $\mathrm{mL}$ e multiplica-se pela densidade do solvente utilizado. Assim: $\mathrm{m}=$ [volume injetado $(\mathrm{mL}) \times$ densidade do solvente $\left.\left(\mathrm{g} \mathrm{mL}^{-1}\right)\right] ; \mathrm{R}=$ constante de proporcionalidade $=8,31 \mathrm{x}$ $10^{6}\left(\mathrm{~Pa} \mathrm{~mL} \mathrm{~mol}^{-1} \mathrm{~K}^{-1}\right) ; \mathrm{T}=$ temperatura absoluta do injetor $(\mathrm{K})$, obtida por $\mathrm{K}={ }^{\circ} \mathrm{C}+273$; $\mathrm{p}=$ pressão absoluta no injetor, em Pa. A pressão informada pelo equipamento $\left(\mathrm{p}_{\mathrm{eq}}\right)$ é uma pressão relativa, adicional à pressão atmosférica $\left(\mathrm{p}_{\mathrm{atm}}\right)$. Esta pressão, expressa em $\mathrm{Pa}$, deve ser somada à pressão atmosférica na mesma unidade; $\mathrm{M}=$ massa molar do solvente, em $\mathrm{g} \mathrm{mol}^{-1}$.

\section{Terceira etapa}

Realização das análises com cada uma das condições estabelecidas e avaliação dos resultados de 8 compostos representativos, com diferentes valores de $t_{R}$ conforme Tabela 1 . Para o cálculo da área normalizada foi considerada a maior área para cada um dos compostos como $100 \%$ e outros valores expressos como porcentagens relativas ao melhor resultado. Os compostos, preparados em acetato de etila, foram avaliados em concentrações de 25 ou $40 \mu \mathrm{g} \mathrm{L}^{-1}$, com exceção do padrão interno que foi avaliado em $500 \mu \mathrm{g} \mathrm{L}^{-1}$.

\section{Quarta etapa}

Construção de modelos quadráticos utilizando como variáveis independentes os valores de pressão, temperatura e volume de injeção de cada um dos experimentos da Tabela 2, e como variáveis dependentes as respostas de intensidade de sinal dos compostos de diferentes características físico-químicas (volatilidades, polaridade, estabilidade, etc.) e, consequentemente, diferentes tempos de retenção, para avaliar o comportamento das respostas em função dos parâmetros de otimização e a dependência ou não das características de retenção.

\section{Quinta etapa}

Avaliação dos resultados através de gráficos da intensidade do sinal obtida para cada volume de expansão, tendo como referência o volume de backflush do injetor.

\section{RESULTADOS}

A Tabela 2 apresenta os volumes de gás gerado a partir das condições cromatográficas e as variáveis independentes de cada um dos experimentos do planejamento. Além disso, mostra as médias e os desvios padrão $(n=8)$ das áreas normalizadas dos picos para todos os compostos avaliados. As áreas dos picos são proporcionais às quantidades dos analitos que atingem o detector apresentando uma relação direta com o volume de gás, que é efetivamente transferido do sistema de injeção splitless para a coluna cromatográfica.

Os maiores sinais de resposta foram obtidos com volumes de gás de 488 e $497 \mu \mathrm{L}$, muito próximos ao volume efetivo do insersor, que é de $490 \mu \mathrm{L}$. A utilização de pressões elevadas possibilita a injeção de volumes maiores sem a ocorrência de uma grande expansão do gás gerado pela vaporização da solução injetada. Ou seja, este fenômeno pode ser claramente entendido pela lei dos gases ideais e verificado utilizando-se um planejamento experimental rápido e simples.

Analisando a Tabela 2 observa-se que as condições cromatográficas que geraram volumes superiores $(582$ e $856 \mu \mathrm{L})$ produziram um decréscimo na sensibilidade, devido à perda de parte do volume do solvente com os analitos através da purga de septo. Nos casos onde os volumes de gás foram menores que o volume efetivo do insersor, os resultados observados foram piores em função dos volumes de injeção. Assim, a melhor situação é obtida com a utilização de maiores volumes de injeção e pressões suficientemente elevadas que garantam um volume de gás próximo ao volume de backflush. A Figura 1a destaca o comportamento dos compostos avaliados através do modelo quadrático que relaciona as duas variáveis independentes, volume injetado e pressão no injetor, com a sensibilidade do sistema. De acordo com a Figura 1a é possível verificar um compromisso entre a pressão no injetor e o volume injetado. No entanto, a Figura $1 \mathrm{~b}$ destaca que a temperatura não é um fator determinante, podendo ser controlada de acordo com outros interesses, tais como: possibilidade de ocorrer degradação de analitos termo-instáveis ou melhoria na transferência de compostos menos voláteis. Da mesma forma, a Figura 1c confirma a baixa influência da temperatura na intensidade do sinal analítico. Os resultados dos gráficos da Figura 1 sugerem que o sistema não apresenta uma condição ótima teórica, já que não há estabilização da 


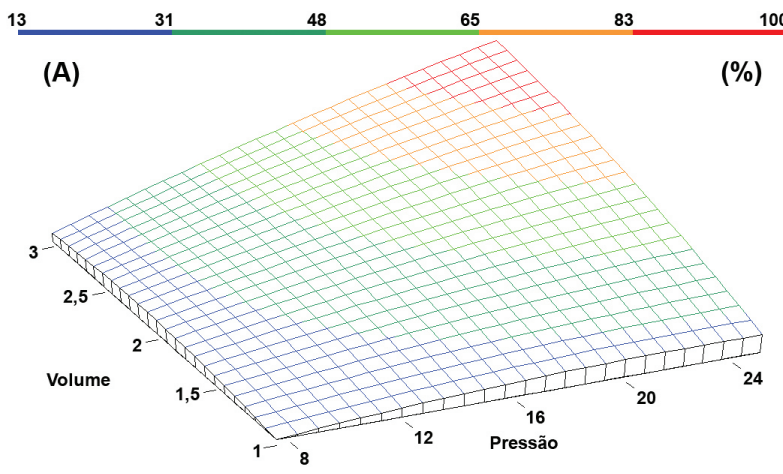

\begin{tabular}{llllll}
22 & 32 & 42 & 53 & 63 & 74 \\
\hline
\end{tabular}

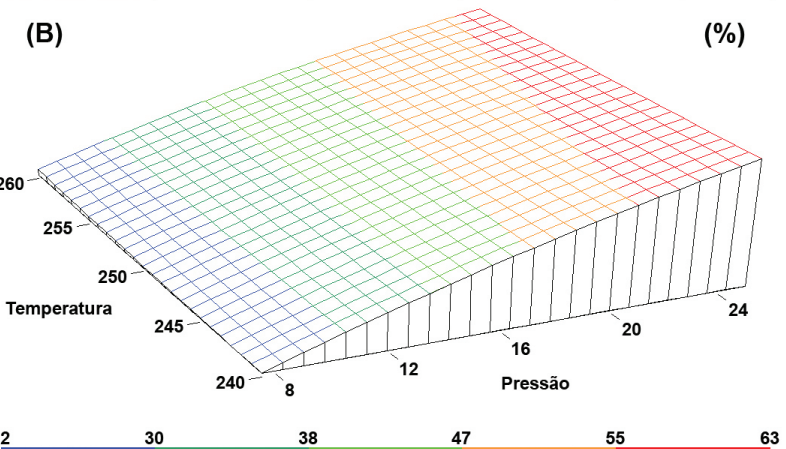

(C)

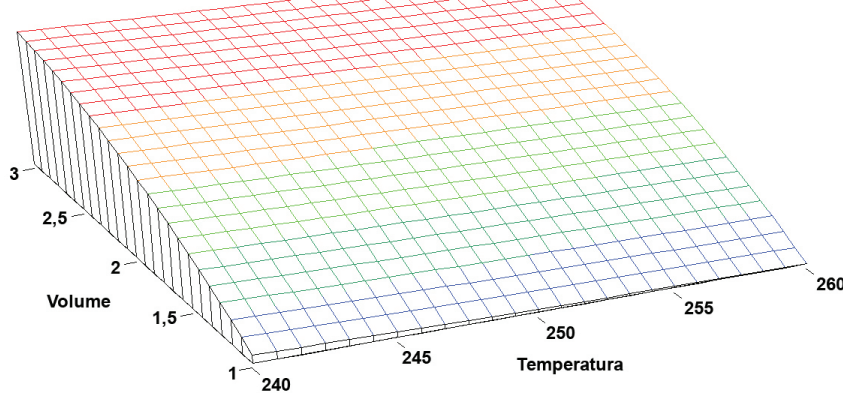

Figura 1. Área normalizada obtida em relação ao (a) volume injetado e a pressão no injetor, (b) temperatura e pressão no injetor e (c) volume injetado e temperatura no injetor

intensidade do sinal analítico em altas pressões e volumes. De fato, a lei dos gases estabelece que quanto maior a pressão no injetor, menor será o volume de gás gerado e, consequentemente, maior será o volume de injeção permitido no sistema splitless. Contudo, a decisão do limite de pressão utilizado depende das limitações instrumentais ou metodológicas, como a alteração em tempos de retenção.

A Figura 2 mostra um gráfico das médias da intensidade dos sinais (área dos picos em relação à maior área), com os respectivos desvios padrão, para os compostos avaliados obtidas através dos resultados da Tabela 2. Os melhores resultados foram obtidos empregando-se as seguintes condições: injeção de $3 \mu \mathrm{L}$, modo splitless pulsado com 25 psi, que geram volumes de gás comparáveis ao volume de backflush ${ }^{15}$ Nesse estudo, foi observado que volumes de gás acima do volume efetivo do insersor reduzem fortemente a intensidade do sinal para cerca de $20 \%$ da intensidade obtida com as condições otimizadas. Com a etapa de otimização (splitless pulsado e maior volume de injeção) foi possível aumentar a sensibilidade do método quando comparada com as condições usuais (injeção de $1 \mu \mathrm{L}$, temperatura do injetor a $250^{\circ} \mathrm{C}$ e modo splitless com pressão de 7,5 psi), possibilitando diminuir significativamente o fator de pré-concentração e, com isso, a quantidade de amostra que deve ser preparada por extração em fase sólida (SPE), procedimento largamente empregado no preparo

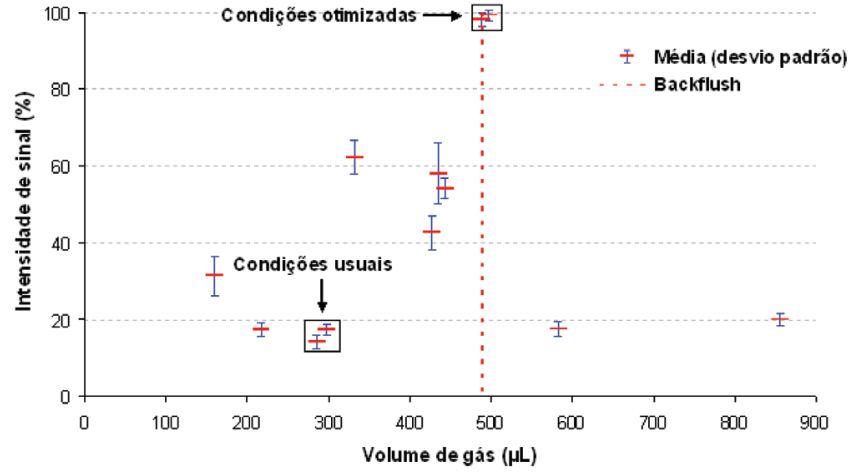

Figura 2. Média da intensidade de sinal versus volume de gás no insersor

de amostras. O aumento de sensibilidade, a diminuição no tempo de execução da etapa de pré-concentração e, consequentemente, dos custos de análise são fatores relevantes alcançados neste trabalho.

Para a verificação prática dos parâmetros propostos, é fundamental que a temperatura inicial da programação do forno da coluna seja mantida suficientemente baixa (aproximadamente $30{ }^{\circ} \mathrm{C}$ abaixo do ponto de ebulição do solvente) para garantir o efeito solvente, evitando possíveis deslocamentos nos tempos de retenção dos compostos nas diferentes condições avaliadas. Caso contrário, será necessário alterar as janelas de tempo empregadas no monitoramento seletivo de íons do espectrômetro de massas. ${ }^{12}$

As condições do sistema de injeção do GC-MS podem ser otimizadas a partir do procedimento descrito, permitindo obtenção de uma maior sensibilidade no desenvolvimento de um método multirresíduo de pesticidas em água potável. $\mathrm{O}$ aumento da resposta analítica foi confirmado para todos os pesticidas e padrões avaliados. Neste trabalho não foi realizado um estudo com matriz da amostra. No entanto, o fenômeno físico que rege o volume gasoso depende somente das variáveis contidas na equação de estado de Clapeyron. Por esta razão, se o volume de solvente é muito maior que a soma dos analitos mais a matriz da amostra (e isso é verdade para análise de traços), o volume de solvente será o responsável pelo volume total de gás formado.

\section{CONCLUSÕES}

Este estudo mostrou a possibilidade de aplicação direta das leis dos gases ideais para a otimização da resposta cromatográfica. Esta abordagem deve buscar solventes com maior massa molar possível (menor coeficiente de expansão térmica, segundo teoria dos gases ideais) dentro do contexto de solubilidade, toxicidade e custos (parâmetros que devem ser avaliados de acordo com as necessidades do laboratório). Outros fatores, como a escolha de colunas com pequeno diâmetro e grande comprimento, contribuem para a possibilidade de aumento de pressão no injetor em baixa vazão. Esta observação, aliada ao modo de injeção pulsado, viabiliza a utilização de volumes de injeções maiores. Da mesma forma, insersores com grande volume efetivo são preferidos (por aumentar a capacidade efetiva de transferência de amostra). Observando estes parâmetros teóricos simples, pode-se aumentar em várias vezes a intensidade do sinal analítico, diminuindo a necessidade de elevados fatores de pré-concentração na etapa de preparo de amostra. Por fim, o sistema pode ser rapidamente verificado aplicando-se um planejamento simples como o que foi exposto permitindo, assim, a visualização da resposta instrumental em função dos volumes de expansão do solvente obtido pela equação de Clapeyron. Embora este trabalho tenha mostrado basicamente a verificação de parâmetros teóricos como auxílio para tomada de decisões envolvendo otimização de sensibilidade, outras possibilidades, como estudo de degradação, velocidade de transferência 
do analito e estudos de repetitividade, podem ser realizados também sob este ponto de vista.

\section{AGRADECIMENTOS}

Ao CNPq, à CAPES e à FINEP pelos apoios financeiros concedidos.

\section{REFERÊNCIAS}

1. Council Directive 98/83/EC of November 3, 1998 on the Quality of Water Intended for Human Consumption; Official Journal of the European Communities, 1998, L 330.

2. Brasil, Ministério da Saúde; Portaria $n$. $^{\circ} 518$, de 25/3/2004, disponível em http://portal.saude.gov.br/portal/arquivos/pdf/portaria_518_2004.pdf, acessada em Maio 2010.

3. Brasil, CONAMA; Resolução no 357, de 17/3/2005, disponível em www. mma.gov.br/port/conama/res/res05/res35705.pdf, acessada em Maio 2010.

4. Qiu, C.; Cai, M.; J. Chromatogr., A 2010, 1217, 1191.

5. Khalili-Zanjani, M. R.; Yamini, Y.; Yazdanfar, N., Shariati, S.; Anal. Chim. Acta 2008, 606, 202.

6. Filho, A. M.; dos Santos, F. N.; Pereira, P. A. de P.; Microchem. J. 2010, 96, 139.

7. van der Lee, M. K.; van der Weg, G.; Traag, W. A.; Mol, H. G. J.; J. Chromatogr., A 2008, 1186, 325.

8. Nguyen, T. D.; Lee, K. J.; Lee, M. H.; Lee, G. H.; Microchem. J. 2010, 95, 43.

9. Fernandez-Alba, A.; Chromatographic-Mass Spectrometric Food Analysis for Trace Determination of Pesticide Residue, Elsevier: Almeria, 2005, p. 487.

10. Reeve, R. N.; Introduction to Environmental Analysis, John Willey \& Sons: West Sussex, 2002, p. 78-104.

11. Primel, E. G.; Zanella, R.; Kurz, M. H. S.; Gonçalves, F. F.; Machado, S. L. O.; Marchezan, E.; Quim. Nova 2005, 28, 605.

12. Wells, M. J. M. Em Principles of Extraction and the Extraction of Semivolatile Organics from Liquids; Mitra, S., ed.; John Wiley \& Sons, Inc.: Hoboken, 2003, vol. 162, p. 1-138.
13. Nevado, J. J. B.; Cabanillas, C. G.; Llerena, M. J. V.; Robledo, V. R.; Microchem. J. 2007, 87, 62.

14. Sabin, G. P.; Dissertação de Mestrado, Universidade Federal de Santa Maria, Brasil, 2007.

15. Busch, M.; Stein, G.; Poppitz, W.; Hein, G.; Müller, A.; J. Chromatogr., B: Anal. Technol. Biomed. Life Sci. 2002, 775, 215.

16. Grob, K.; Split and Splitless Injection for Quantitative Gas Chromatography: Concepts, Processes, Practical Guidelines, Sources of Error, Wiley-VCH: Zürich, $4^{\text {th }}$ ed., 2001.

17. Grob, R. L.; Barry, E. F.; Modern practice of gas chromatography, John Wiley \& Sons: New Jersey, $4^{\text {th }}$ ed., 2004.

18. Restek; Operating hints for using split/splitless injectors, Technical Guide, 2001.

19. Kirchner, M.; Matisová, E.; Dömötörová, M.; Zeeuw, J.; J. Chromatogr. A 2004, 1055, 159.

20. Hoh, E.; Mastovska, K.; J. Chromatogr., A 2008, 1186, 2.

21. Murphy, B.; Murphy, C.; Hathaway, B.; A Working Method Approach for Introductory Physical Chemistry Calculations: Numerical and Graphical Problem Solving, The Royal Society of Chemistry Paperbacks: Cambridge, 1997.

22. Atkins, P.; Paula, J.; Físico-Química, $8^{\mathrm{a}}$ ed., LTC: Rio de Janeiro, 2008, vol. 1.

23. Sabin, G. P.; Prestes, O. D.; Adaime, M. B.; Zanella, R.; J. Braz. Chem. Soc. 2009, 20, 918

24. Doherty, L.; Optimizing the Agilent Technologies 6890 Series GC for High Performance MS Analysis, Technical Overview, 5988-9944EN (14-11-2003), p. 1-10.

25. Mastovska, K.; Lehotay, S. J.; J. Chromatogr., A 2003, 1000, 153.

26. Tomlin, C. D. S.; The e-Pesticide Manual, $13^{\text {th }}$ Ed., versão 3.0, Londres, 2003-2004.

27. Klee, M. S.; GC Inlets - An Introduction, Agilent Technologies, Inc.: Wilmington, 2005.

28. de Barros Neto, B. ; Scarminio, I. S.; Bruns, R. E.; Como fazer experimentos: pesquisa e desenvolvimento na ciência e na indústria, Edit. da Unicamp: Campinas, 2007. 